\title{
Latest Higgs results from ATLAS
}

\author{
E. Rossi* \\ On behalf of ATLAS Collaboration \\ Università degli Studi di Napoli "Parthenope" and INFN \\ E-mail: elvira.rossiecern.ch; elly@na.infn.it
}

\begin{abstract}
An overview of the Higgs boson physics program performed in the ATLAS experiment at LHC using the entire set of Run I data collected in 2011 and 2012 is presented. Emphasis is given to recent measurements on the production and decay properties of the Higgs boson. No indications for deviations from the Standard Model (SM) nature of the Higgs boson are observed.
\end{abstract}

XXIII International Workshop on Deep-Inelastic Scattering,

27 April - May 12015

Dallas, Texas

* Speaker. 


\section{Introduction}

On 4th July 2012 both the ATLAS and CMS Collaborations [1] announced the discovery of a Higgs-like particle with mass around $125 \mathrm{GeV}$ [2]. As more data were collected, the properties (spin, CP, couplings, cross section) of the discovered particle were measured. LHC has been operated at center-of-mass energies $(\sqrt{s})$ of $7 \mathrm{TeV}$ in 2011 and of $8 \mathrm{TeV}$ in 2012 (Run I). After a period dedicated to the upgrade of the detectors and the experimental settings, now the Run II phase is starting with $\sqrt{s}=13 \mathrm{TeV}$. The ATLAS detector has performed very efficiently during Run I, having collected data corresponding to an integrated luminosity of $20.4 \mathrm{fb}^{-1}$ at $\sqrt{s}=8 \mathrm{TeV}$ and $4.6 \mathrm{fb}^{-1}$ at $\sqrt{s}=7 \mathrm{TeV}$. This paper summarises recent experimental results by ATLAS on the search of a SM-like Higgs boson and the determination of its properties.

\section{Higgs searches in bosonic decay modes: $H \rightarrow Z Z^{*}, H \rightarrow \gamma \gamma$ and $H \rightarrow W W^{*}$}

\section{$H \rightarrow Z Z^{*} \rightarrow 4 \ell(\ell=e$ or $\mu)$ decay channel}

The bosonic decays were used as a tool for the discovery at the initial stage and subsequently as a tool for the measurement of the properties and the confirmation of the discovery. In all these channels the production is studied both inclusively and with events categorised according to the characteristics of the different production modes. The $H \rightarrow Z Z^{*} \rightarrow 4 \ell$ channel has a small rate but produces a very clean final state with full reconstruction of the Higgs boson so it's ideal for studies concerning the Higgs boson properties. The final state consists of four isolated leptons (electrons or muons) originating from the primary vertex, one pair of which is expected to have an invariant mass consistent with the $Z$ boson mass. The $Z Z^{*}$ continuum production is the irreducible background. The reducible background consists of $Z$ plus jets and $t \bar{t}$ production and it is estimated using data driven techniques. Events are categorised in subchannels according to the flavour composition of the pairs. Further categorisation of the events, in order to distinguish the different production modes, proceeds according to the jet content of the events and the existence of additional leptons. Figure 1(a) shows the four-ton invariant mass distribution of the candidate events. Details of recent ATLAS results can be found in Ref. [3].

\section{$H \rightarrow \gamma \gamma$ decay channel}

The branching ratio of the $H \rightarrow \gamma \gamma$ channel is small, but it produces a clean final state with full reconstruction of the Higgs boson and a signal $m_{\gamma \gamma}$ peak on top of a large but smooth background distribution. It is therefore an excellent channel to measure the mass and couplings of the Higgs boson. The final state consists of two isolated photons originating from the primary vertex. The diphoton continuum production constitutes the dominant (80\%) irreducible background, while the reducible background consists of g-jet and jet-jet. Categories aiming to distinguish events according to the production mechanism of the Higgs boson are formed. Furthermore, events are categorised according to the transverse momentum of the diphoton system and the pseudorapidity of the candidate photons. Figure 1(b) shows the diphoton invariant mass distribution of the candidate events weighted by the signal-to-background ratio in the dataset and category it belongs to. The bottom plot shows the data relative to the background component of the fitted model. The final ATLAS analysis of Run I results are summarised in Ref. [4]. 


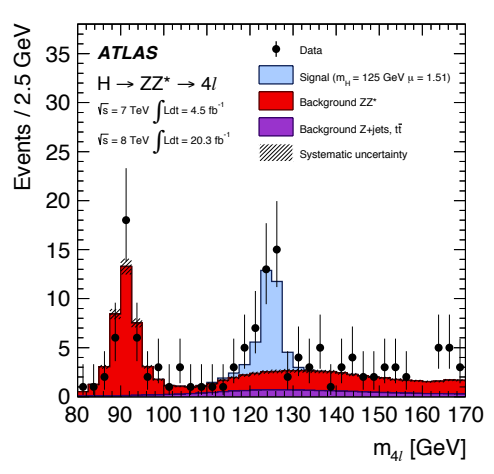

(a)

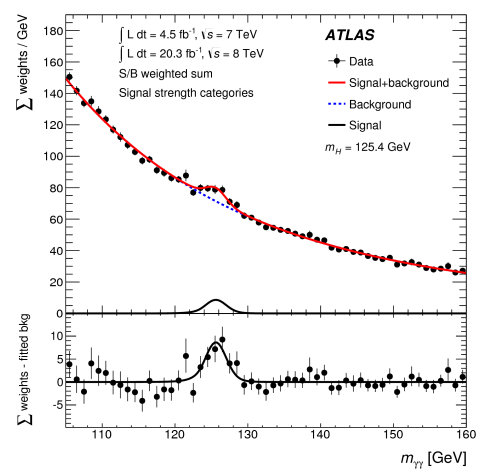

(b)

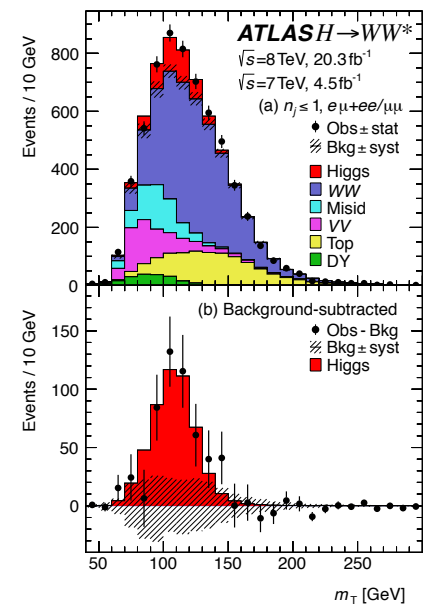

(c)

Figure 1: (a) Four-lepton invariant mass distribution [3]; (b) diphoton invariant mass distribution (top) and the data relative to the background component (bottom) [4]; (c) transverse mass distributions for $n_{j} \leq 1$ for the three lepton-flavour samples (top) and the residuals of the data respect to background in $H \rightarrow W W^{*}$ (bottom) [5].

\section{$H \rightarrow W W^{*} \rightarrow \ell v \ell v(\ell=e$ or $\mu)$ decay channel}

The $H \rightarrow W W^{*}$ decay mode has a large branching ratio. However, the cleaner way to observe this channel is through $H \rightarrow W W^{*} \rightarrow \ell v \ell v$, where both the $\mathrm{W}$ bosons decay leptonically to electrons or muons. Therefore, due to the two missing neutrinos the Higgs final state can not be reconstructed completely and the signal appears as a broad peak in the transverse mass $m_{T}$ distribution. The signal consists of two isolated, high $p_{T}$, opposite sign leptons plus missing energy. The main irreducible background is the $W W^{*}$ continuum production. Many sources contribute to the reducible one, such as single top, $t \bar{t}, W / Z$ plus jets and other diboson production. Events are categorised in subchannels according to the flavour composition of the lepton pair and the number of reconstructed jets in the event. Further categorisation of the events, to distinguish the Vector Boson Fusion production mode, is performed using a boosted decision tree multivariate method. A likelihood function is constructed to simultaneously model the yields of the various subsamples in both the signal and the various control regions. Figure 1 (c) shows the post-fit combined transverse mass distributions for $n_{j} \leq 1$ for the three lepton-flavour samples. The bottom plot shows the residuals of the data with respect to the estimated background compared to the expected distribution for a SM Higgs boson with $m_{H}=125 \mathrm{GeV}$. Final results of the Run I analysis are presented in Ref. [5].

\section{Higgs searches in fermionic decay modes: $H \rightarrow b \bar{b}, H \rightarrow \tau^{+} \tau^{-}$and $H \rightarrow \mu^{+} \mu^{-}$}

\section{$\underline{H \rightarrow b \bar{b}}$}

The only way to probe the Higgs boson top quark Yukawa coupling is through its associated production $t \bar{t} H$. This decay channel has the largest branching ratio (BR) of $58 \%$ at $m_{H}=125 \mathrm{GeV}$. The $H \rightarrow b \bar{b}$ final state suffers from huge QCD multijet backgrounds, making impossible the tagging of this final state alone. The only feasible way for this measurement, is through the use of associated 
production with a vector boson or a $t \bar{t}$ pair. The analysis is categorised according to the number of

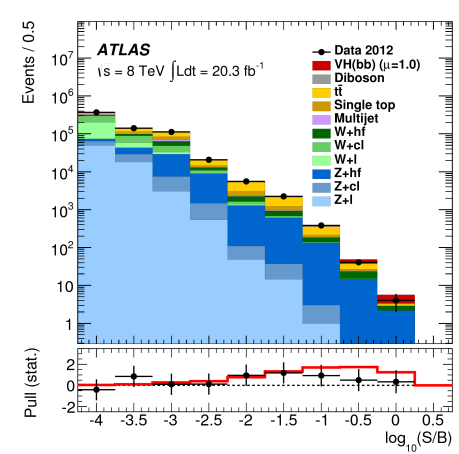

(a)

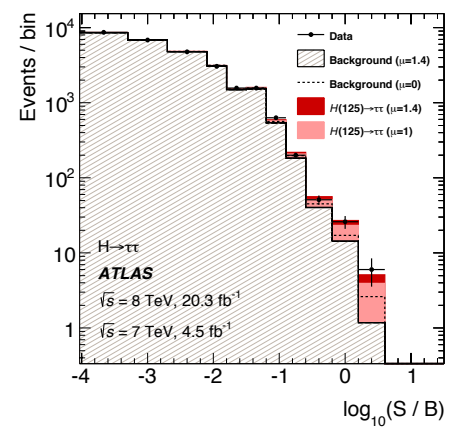

(b)

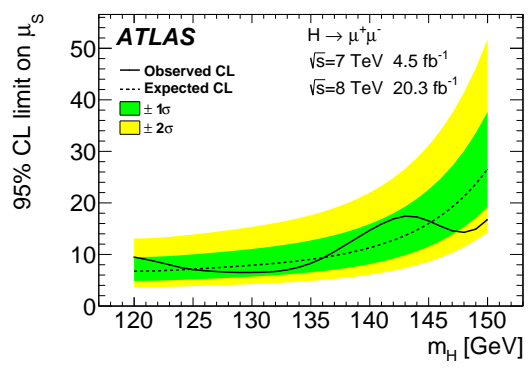

(c)

Figure 2: Event yields as a function of $\log (\mathrm{S} / \mathrm{B})$ for data, background and Higgs boson signal with $m_{H}=$ $125 \mathrm{GeV}$ for the $8 \mathrm{TeV}$ data for $H \rightarrow b \bar{b}$ [6] (a) and for $H \rightarrow \tau^{+} \tau^{-}$analysis [7] (b). (c) Observed (solid) and expected (dashed) $95 \% \mathrm{CL}$ upper limits on the $H \rightarrow \mu^{+} \mu^{-}$signal strength as a function of $m_{H}$ [8].

leptons in the final state and on the $p_{T}$ of the vector boson. Figure 2 (a) shows the combined results from all the analysis categories. An excess is observed (expected) at the $1.4 \sigma(2.6 \sigma)$ level over the background-only hypothesis. Details of the search for $H \rightarrow b \bar{b}$ decays can be found in Ref. [6].

\section{$\underline{H \rightarrow \tau^{+} \tau^{-}}$}

More favorable signal-to-background conditions are expected for $H \rightarrow \tau^{+} \tau^{-}$decays that has a BR of $6.3 \%$ at $m_{H}=125 \mathrm{GeV}$.Since $\tau$ leptons decay either leptonically or hadronically, the analysis is split into three channels, which are $\tau_{\text {lep }} \tau_{\text {lep }}, \tau_{\text {lep }} \tau_{\text {had }}$, and $\tau_{\text {had }} \tau_{\text {had }}$ where $\tau_{\text {lep }}$ is a leptonic $\tau$-decay object ( $\tau \rightarrow v_{\tau} v_{\ell} \ell$ with $\left.\ell=e, \mu\right)$ and $\tau_{\text {had }}$ is a hadronic $\tau$-decay object ( $\tau \rightarrow v_{\tau}$ hadrons). Figure 2 (b) shows the combined results from all the analysis categories. An excess over the SM background prediction is observed. The probability $p_{0}$ of obtaining a result at least as signal-like as observed in data without signal presence is calculated. For $m_{H}=125.36 \mathrm{GeV}$, the observed $p_{0}$ value is $2.7 \cdot 10^{-6}$, which corresponds to a $4.5 \sigma$ deviation from the background only hypothesis, while $3.4 \sigma$ deviation is expected. This provides evidence for $H \rightarrow \tau^{+} \tau^{-}$at $m_{H}=125.36 \mathrm{GeV}$. Details of the search for $H \rightarrow \tau^{+} \tau^{-}$decays can be found in Ref. [7].

$\boldsymbol{H} \rightarrow \boldsymbol{\mu}^{+} \boldsymbol{\mu}^{-}$

Since the Higgs boson coupling to fermions is predicted to be proportional to the mass of the corresponding fermions, the branching ratio for this decay is very small $\left(\sim 2 \cdot 10^{-4}\right)$. The signal to background ratio is small as well, $0.2 \%$, but the dimuon spectrum provides a very clean signature with a very good mass resolution and at the same time is the only means of measuring the second generation fermionic couplings. The signal is exactly two isolated opposite sign muons, while there is a huge irreducible background from Drell-Yan. In Figure 2(c) the observed and expected 95\% $\mathrm{CL}$ upper limits on the signal strength as a function of $m_{H}$ over the mass range $120-150 \mathrm{GeV}$ is shown. No excess of events is observed, leading to an observed limit at 95\% CL, of 7.0 times the SM production rate at a Higgs boson mass of $125.5 \mathrm{GeV}$ (with 7.2 expected). Details of the search for this analysis can be found in Ref. [8]. 


\section{Associated top Higgs production}

The production of the Higgs boson in association with a pair of top quarks $(t \bar{t} H)$ provides direct sensitivity to the large Yukawa coupling between the top quark and the Higgs boson.The decay modes of the Higgs boson, $H \rightarrow b \bar{b}, H \rightarrow W^{+} W^{-}, H \rightarrow Z Z, H \rightarrow \tau^{+} \tau^{-}$and $H \rightarrow \gamma \gamma$ give a range of different final states in which to search for $t \bar{t} H$ production. For a Higgs boson with mass $m_{H}=125 \mathrm{GeV}$ the dominant decay mode is into a pair of b-quarks, so it provides the largest number of signal events. However, the large background from the production of $t \bar{t}$ in association with additional jets makes this a challenging signature to separate from the background. Despite the small rate, the diphoton final state provides a clean signal with very good resolution of $m_{\gamma \gamma}$ making this channel promising for the $t \bar{t} H$ search. For the $t \bar{t} H$ search in multi-lepton final states several of the decay modes of the Higgs boson, $H \rightarrow W^{+} W^{-}, H \rightarrow Z Z, H \rightarrow \tau^{+} \tau^{-}$, have been studied. These signatures have a small SM background and so can be used to search for the $t \bar{t} H$ process. No significant excess of events over the background has been observed in the associated top Higgs production. Details for these analyses can be found in Ref. [9].

\section{Higgs properties}

After the discovery, the ATLAS collaboration has put great effort to study the properties of the newly discovered boson. In order to verify the SM origin or not of the Higgs boson, detailed measurements of its mass, couplings, spin, parity, width and cross section have been performed.

Mass: In the SM, the Higgs boson mass is not predicted, so its measurement is therefore required for precise calculations of electroweak observables. The $H \rightarrow \gamma \gamma$ and $H \rightarrow Z Z^{*} \rightarrow 4 \ell$ channels are most sensitive to the Higgs boson mass measurement. The Higgs boson produces a narrow mass peak with a typical experimental resolution of $1.6 \mathrm{GeV}$ to $2 \mathrm{GeV}$ over a smooth background. Several improvements have been incorporated in the final ATLAS analysis of Run I data, that affect directly the determination of the Higgs boson mass [10]. The measured Higgs boson mass in the $H \rightarrow \gamma \gamma$ channel is: $m_{H}=125.98 \pm 0.42$ (stat) \pm 0.28 (syst) $\mathrm{GeV}$ and in the $H \rightarrow Z Z^{*} \rightarrow 4 \ell$ channel is: $m_{H}=124.51 \pm 0.52$ (stat) \pm 0.06 (syst) GeV. The combined mass measurement result is: $m_{H}=125.36 \pm 0.37$ (stat) \pm 0.18 (syst) $\mathrm{GeV}$. The consistency between the individual measurements of the Higgs boson mass in the two channels is of $4.9 \%$, corresponding to $1.97 \sigma$. A combined mass measurement with CMS data has been performed and recently published [10].

Couplings: Details of the combined analyses of the Higgs boson production and decay rates as well as its coupling strengths to vector bosons and fermions can be found at Ref. [11]. The combinations take inputs from all the analyses presented in the previous sections. Figure 3 (a) shows the updated measurements of the signal-strength parameter from a simultaneous fit to all decay channels that were analysed. The overall signal strength of each analysis is the combined result of the measurements for different production processes. The measurements are consistent and compatible with a single value with a probability of $76 \%$. They have been combined to result in a global signal-strength value of [11]: $\mu=1.18_{-0.14}^{+0.15}=1.18 \pm 0.10($ stat $) \pm 0.07$ (expt $)_{-0.07}^{+0.08}$ (theo) consistent with the SM expectation of $\mu=1$ with a probability of $18 \%$. The uncertainty on the combination 


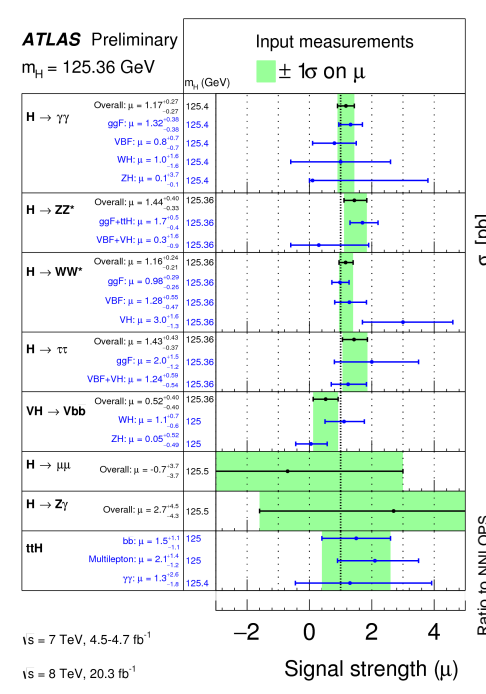

(a)

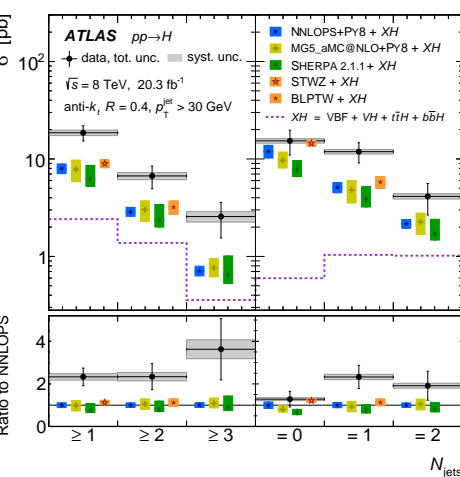

(b)

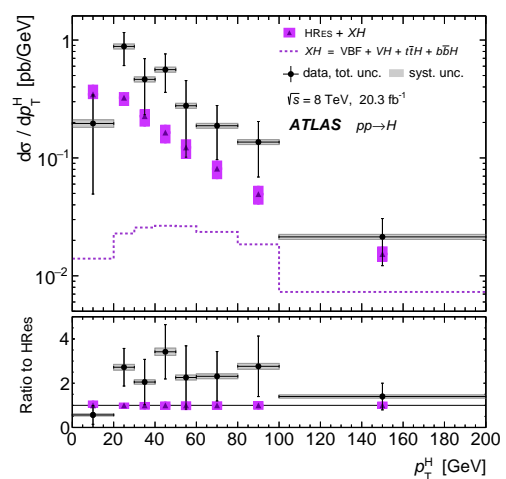

(c)

Figure 3: (a) Summary of the signal strength measurements from individual analyses [11]; (b) Measured Higgs boson production cross sections in inclusive and exclusive jet multiplicity bins compared to different theoretical predictions [12]; (c) Differential cross sections for inclusive Higgs boson production with the respect to Higgs boson transverse momentum [12].

has comparable statistical and systematic components.

Cross-section: ATLAS measured total and differential cross sections for Higgs boson production using the $H \rightarrow \gamma \gamma$ and $H \rightarrow Z Z^{*} \rightarrow 4 \ell$ events at $\sqrt{s}=8 \mathrm{TeV}$ [13]. The measured cross sections account for detector efficiency, fiducial acceptances and branching fractions. Figure 3 (b) shows the measured Higgs boson production cross sections in inclusive and exclusive jet multiplicity bins compared to different theoretical predictions. The measured total cross section is $33.0 \pm 5.3$ (stat) \pm 1.6 (sys) pb which is higher than NNLO and N3LO calculations [12], but still consistent with the theory predictions. Figure 3 (c) shows the differential cross section as a function of the Higgs boson transverse momentum $p_{T}^{H}$.

Spin-Parity: The study of the spin and parity properties of the Higgs boson in ATLAS is based on the $H \rightarrow \gamma \gamma, H \rightarrow Z Z^{*} \rightarrow 4 \ell$ and $H \rightarrow W W^{*} \rightarrow \ell \nu \ell v$ decay channels and their combination. It relies on discriminant observables chosen to be sensitive to the spin and parity of the signal while preserving the discrimination against the various backgrounds. Recent ATLAS studies can be found in Ref. [14]. The SM Higgs boson hypothesis, corresponding to the quantum numbers $J^{P}=0^{+}$, is tested against several alternative spin models. Three possible scenarios for the spin and parity of the boson are considered: the hypothesis that the observed resonance is a spin-2 resonance, a pure spin-0 CP-even or CP-odd BSM Higgs boson, or a mixture of spin-0 CP-even and CP-odd states. An example of distributions of the test statistic used to derive the results is presented in Figure 4 (a). The combination of the three decay processes allow the exclusion of all considered non-SM spin models at more then $99 \% \mathrm{CL}$ in favour of the SM spin-0 hypothesis. For spin 0 models, 


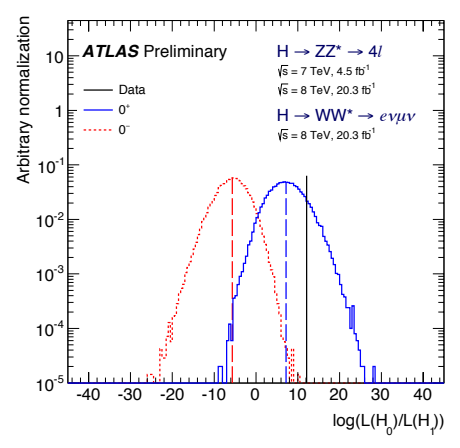

(a)

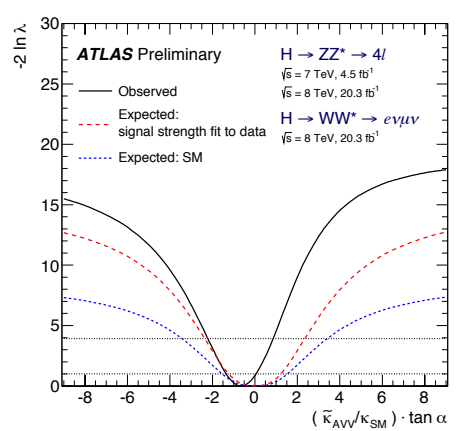

(b)

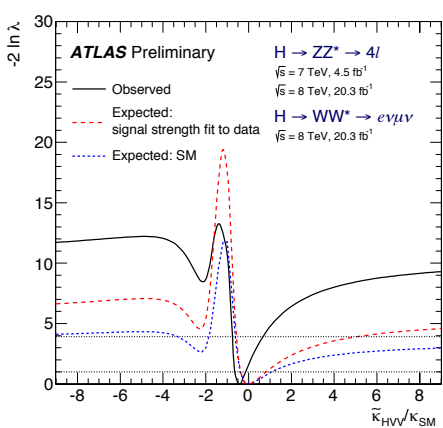

(c)

Figure 4: (a) Example of distribution of the test statistic in the pseudoscalar hypothesis [14]; Likelihood scan as a function of BSM CP-odd (b) and CP-even (c) coupling parameters [14].

some CP-mixing scenarios in the Higgs sector have been considered using the $H \rightarrow Z Z^{*} \rightarrow 4 \ell$ and $H \rightarrow W W^{*} \rightarrow \ell v \ell v$ events. Figures 4 (b) and (c) show likelihood scan as a function of BSM CPodd and CP-even coupling parameters. The observed results are consistent with no BSM coupling, and no $\mathrm{CP}$ violation in the Higgs sector is observed within the current precision.

\section{References}

[1] ATLAS Collaboration, JINST 3 (2008) S08003; CMS Collaboration, JINST 3 (2008) S08004.

[2] ATLAS Collaboration, Phys. Lett. B 716 (2012) 1; CMS Collaboration, Phys. Lett. B 716 (2012) 30.

[3] ATLAS Collaboration, Phys. Rev. D. 91, 012006 (2015).

[4] ATLAS Collaboration, Phys. Rev. D. 90, 112015 (2014).

[5] ATLAS Collaboration, arXiv:1412.2641 (submitted to Phys. Rev. D).

[6] ATLAS Collaboration, JHEP 01 (2015) 069.

[7] ATLAS Collaboration, JHEP 04 (2015) 117.

[8] ATLAS Collaboration, Phys. Lett. B 738 (2014) 68-86.

[9] ATLAS Collaboration, Phys. Lett. B 740 (2015) 222-242; ATLAS-CONF-2015-006 http://cds.cern.ch/record/2002125; arXiv:1503.05066 (accepted by EPJ).

[10] ATLAS Collaboration, Phys. Rev. D. 90, 052004 (2014); ATLAS and CMS Collaborations, Phys. Rev. Lett. 114, 191803.

[11] ATLAS collaboration, ATLAS-CONF-2015-007, http://cds.cern.ch/record/2002212.

[12] LHC Higgs cross section working group, arXiv:1101.0593; Anastasiou et al. Phys. Rev. Lett. 114, 212001 (2015).

[13] ATLAS Collaboration, arXiv:1504.05833, submitted to PRL.

[14] ATLAS Collaboration, ATLAS-CONF-2015-008 (2015), http://cds.cern.ch/record/2002414. 